that the ferromagnet has above the critical point. But theories that govern phase transitions in cosmology and in condensedmatter physics are essentially identical, so experiments on cosmology can in theory be performed in a laboratory.

Common to cosmology and condensedmatter physics is the question, how big are the pieces of broken-symmetry vacuum just after the phase transition? The size of such coherent domains manifests itself in the initial density of topological defects. These are intrusions of the old symmetric vacuum, imprisoned by configurations of the new broken-symmetry state that are impossible to disentangle. For example, when symmetry breaking involves two distinct choices, at a boundary between the two such regions there will be a domain wall (Fig. 1). One can expect approximately one 'unit' of topological defect - one monopole, a domain-sized piece of a string, or a 'tile' of a domain wall - per domain ${ }^{3}$, so the initial density of defects gives the size of the pieces of broken symmetry.

Superfluids are an excellent example of symmetry breaking that leads to topological defects. They are described by a complex 'order parameter', $\psi=|\psi| \mathrm{e}^{i \theta}$, a wave function of the Bose condensate of helium atoms. In some ways, $\psi$ can be thought of simply as a probability wave familiar from ordinary quantum mechanics. The square of its absolute value gives the density of the superfluid, which in a sense is the probability of finding an atom of superfluid at a particular point. But the analogy is incomplete. The wavefunction of the superfluid Bose condensate does not satisfy the superposition principle, and its square is not conserved. Moreover, in helium-3 the order parameter $\psi$ is not just a complex number, but a tensor, which describes rotational states of the pairs of ${ }^{3} \mathrm{He}$ atoms (individual ${ }^{3} \mathrm{He}$ atoms are fermions, and like electrons in a superconductor they must pair before they can Bosecondense).

Symmetry breaking is induced by a change in the shape of the effective potential. Above the critical temperature, the potential minimum is at the origin and $\psi$ fluctuates near there (Fig. 2), but below the critical point the potential has a minimum away from the origin, along a rim of the 'sombrero'. To settle there, $\psi$ has to break symmetry it has to choose a value of $\theta$.

Topological defects in superfluids arise when the phase, $\theta$, changes continuously by $2 \pi$ along a closed path in space - then there must be a point inside the loop where $\theta$ is undefined. The arrows representing the order parameter will all be pointing either directly towards or away from the "vortex

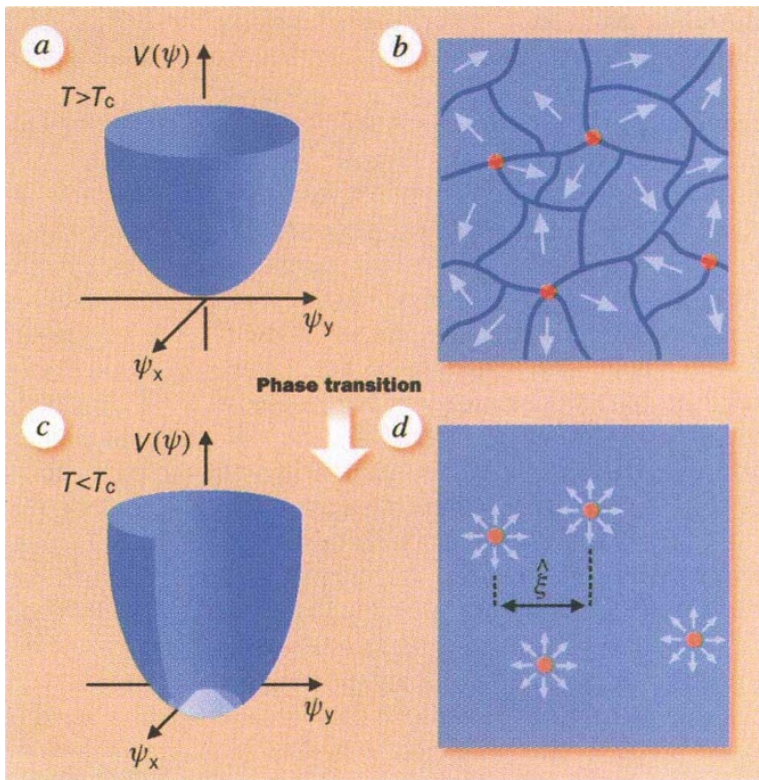

FIG. 2 Dynamics of defect formation in superfluids. $a, b$, Above the phase transition, the two-dimensional (complex) order parameter $\psi$ fluctuates around the minimum of the potential, assuming values which point in approximately the same direction (or phase, $\theta$ ) within each local domain. $c, d$, When this configuration is frozen out by the phase transition, the symmetric phase will be trapped in some locations: these are defects. In superfluids, such defects are lines of vortical flow. Their energy is related to the difference in potential energies between the hill at $\psi=0$ and the minimum along the circle of broken-symmetry configurations. It can also be regarded as the energy of rotation of the vortex.

\section{To derive the density of defects:}

Like many of the fundamental fields in cosmology, superfluids undergo what are called second-order phase transitions. As such a system approaches a transition infinitesimally slowly, its order parameter becomes correlated over larger and larger domains. The domain size is characterized by the correlation length $\xi$, which, in equilibrium, increases and diverges to infinity as the temperature approaches the critical temperature $T_{c}$. More precisely, in ${ }^{3} \mathrm{He}$ and many other systems $\xi=\xi_{0} \epsilon^{-1 / 2}$, where $\xi_{0}$ is a constant and $\epsilon$ is the relative temperature, $\epsilon=\left(T-T_{\mathrm{c}}\right) / T_{\mathrm{c}}$. So, if the temperature changes slowly enough through $T_{c}, \xi$ diverges to infinity, and in any finite volume there will be no defects. However, it also takes an increasingly long relaxation time $\tau$ for the system to settle to such a uniform equilibrium state, as $\tau=\tau_{0} / \epsilon$. Here, $\tau_{0}$ is the characteristic reaction time far below the phase transition.

If the transition occurs at a finite pace, on a quench timescale $\tau_{Q}$, then $\epsilon=t / \tau_{Q}$, where $t$ is time. There will come a moment $\hat{t}$ when the system will not be able to keep up with the changes in $\epsilon$ - its reaction time will simply be too slow. That will happen when the time remaining until the phase transition is equal to its reaction time, $\tau(\hat{t})=\hat{t}$ or $\hat{t}=\tau_{0} \tau_{Q} / \hat{t}$. Hence, $\hat{t}=\left(\tau_{0} \tau_{Q}\right)^{1 / 2}$. Before the critical temperature is reached, the state of the system will fall out of step with what the equilibrium should be, and by the time its reflexes are recovered it will be already in the broken-symmetry state. The correlation length corresponding to $\hat{t}$ should then be the size of the domains ${ }^{4}$, the 'effective causal horizon' for the order parameter. That is, the size of the uniform domain $\hat{\xi}=\xi_{0} \hat{\epsilon}^{-1 / 2}=\xi_{0}\left(\tau_{Q} / \tau_{0}\right)^{1 / 4}$ defines the extent of the region within which the order parameter has managed to agree on how to break the symmetry. Vortex lines in ${ }^{3} \mathrm{He}$ should form with a density of about one $\hat{\xi}$ of length per $\hat{\xi}^{3}$ volume. This is consistent with the numerical simulation ${ }^{10}$ and both the new experiments ${ }^{1,2}$. W.H.Z line', where the field is forced to climb back on top of the sombrero to assume the only value, $\psi=0$, that does not require making a choice of phase. A gradient in $\theta$ necessarily implies a flow, just as an ordinary plane wave flows in the direction of changing phase. In this case the flow pattern is a vortex of superfluid with a non-superfluid core.

The energy corresponding to this rotation was used by the Grenoble group ${ }^{1}$ to detect the presence of vortex lines superfluid analogues of cosmic strings. This can be done by heating up a small volume of superfluid ${ }^{3} \mathrm{He}$ and letting it cool down rapidly, so for a short while the system is in a non-superfluid state. When it returns below the critical temperature, different pieces independently choose the manner in which their symmetry is spontaneously broken. The initial energy used to heat up the fragment of the superfluid above the critical temperature is precisely known, so by monitoring the energy that escapes, one can find out how much of it remains in the vortex lines. The Helsinki group uses an identical procedure to generate defects ${ }^{2}$, but they do it at a somewhat higher temperature, and they use a technique that allows them to count the loops of stringlike vortex lines one by one. In both experiments the initial density of vortex lines is found and used to infer the average size of the like-minded pieces of newly made broken-symmetry superfluid.

The answer confirms a theory of the 